\title{
Singling Out Dynamic and Nondynamic Correlation
}

\author{
Mireia Via-Nadal, ${ }^{\dagger, \ddagger, \|}$ Mauricio Rodríguez-Mayorga, ${ }^{\dagger, \ddagger, \Phi, \|}$

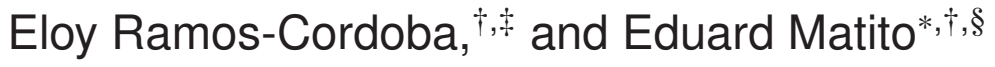 \\ $†$ Donostia International Physics Center (DIPC), 20080 Donostia, Euskadi, Spain \\ $\ddagger$ Kimika Fakultatea, Euskal Herriko Unibertsitatea (UPV/EHU), Donostia, Euskadi, Spain \\ IIInstitut de Química Computacional i Catàlisi (IQCC) and Departament de Química, University \\ of Girona, C/ Maria Aurèlia Capmany, 69, 17003 Girona, Catalonia, Spain \\ §IKERBASQUE, Basque Foundation for Science, 48013 Bilbao, Euskadi, Spain. \\ || Equally contributed \\ E-mail: ematito@gmail.com
}

\begin{abstract}
The correlation part of the pair density is separated into two components, one of them being predominant at short electronic ranges and the other at long ranges. The analysis of the intracular part of these components permits to classify molecular systems according to the prevailing correlation: dynamic or nondynamic. The study of the long-range asymptotics reveals the key component of the pair density that is responsible for the description of London dispersion forces and a universal decay with the interelectronic distance. The natural rangeseparation, the identification of the dispersion forces and the kind of predominant correlation type that arise from this analysis are expected to be important assets in the development of new electronic structure methods in wavefunction, density and reduced density-matrix functional theories.
\end{abstract}




\section{Introduction}

Electron correlation being the holy grail of electronic structure methods, it has been the subject of extended analysis. ${ }^{1-14}$ The solution of quantum many-body problems hinges on the type of correlation present in the system, and one of the most practical classifications consists in the separation between dynamic- and nondynamic-correlation-including methods. Indeed, there are accurate methods to study systems with one predominant correlation type, but systems presenting both correlation types pose one of the greatest current challenges in electronic structure theory. ${ }^{15-17}$

The attempt at taking the best of both worlds has led to a resurgence of interest in hybrid schemes,${ }^{18}$ merging methods that recover different correlation types. ${ }^{19-21}$ Among hybrid implementations, the most successful one is based on the range separation of electron correlation, ${ }^{18,22,23}$ using a mixing function to combine approximations that account for short-range dynamic correlation — such as density functional approximations — with approaches providing correct long-range asymptotics. The performance of these methods pivots on the choice of the function combining the two approaches, which provides a natural splitting of the Coulomb interaction and thus the pair density. ${ }^{24}$ In range-separation approximations, the typical choice is the error function that, in turn, depends on an attenuating parameter, which is both system- and property-dependent. ${ }^{25,26}$ Even though the methods are chosen according to their ability of recovering dynamic and nondynamic correlation, the range-separation of the pair density has not been motivated by the correlation type present in the system, risking double counting of electron correlation.

Thus far, there has been very few attempts to separate dynamic and nondynamic correlation, ${ }^{2,4-9,13,27}$ most of them based on energy calculations. The lack of a physically sound separation of dynamic and nondynamic correlation precludes individual treatment of these effects. We analyze the decomposition of the pair density into three components: the uncorrelated reference and two correlation terms. The latter two behave differently with respect to large changes of the first-order reduced density matrix (1-RDM), permitting the identification of systems with prevalent

dynamic or nondynamic correlation. ${ }^{2,6,7,11,12}$ Some of us have recently used a similar strategy to obtain scalar ${ }^{11}$ and local ${ }^{12}$ measures of dynamic and nondynamic electron correlation from a two- 
electron model. The intracule of the correlation components of the pair density yields a two-fold separation of the Coulomb hole in terms of correlation type and interelectronic range. These components of the pair density display a simpler mathematical form than the total pair density, one of them being dominant at short ranges and one with prevailing long-range contributions. This feature is particularly convenient for the design of energy functionals in wavefunction, density and density matrix functional theories. As a result of this separation, we will clearly identify the part of the pair density that is responsible for the correct description of van der Waals interactions and unveil a universal condition it should satisfy. ${ }^{28}$ To our knowledge, the latter is the only known condition of the pair density that can be employed to design methods including van der Waals interactions.

\section{Theoretical background}

Let us consider the pair density of a $N$-electron system described by the $\Psi(\mathbf{1}, \ldots, \mathbf{n})$ wavefunction,

$$
\rho_{2}(\mathbf{1}, \mathbf{2})=\frac{N(N-1)}{2} \int d_{\mathbf{3}} \ldots d_{\mathbf{n}}|\Psi(\mathbf{1}, \ldots, \mathbf{n})|^{2}
$$

where numerical variables $(\mathbf{1}, \mathbf{2}, \ldots)$ refer to space and spin coordinates. Upon integration over its coordinates, the pair density can be reduced to the intracule density, which only depends on the interelectronic range separation, $s$,

$$
I\left(\rho_{2}, s\right)=\int d_{1} d_{2} \rho_{2}(\mathbf{1}, \mathbf{2}) \delta\left(s-r_{12}\right),
$$

where $r_{12}$ is the Euclidean distance between the electrons at $\mathbf{1}$ and $\mathbf{2}$. The intracule density is the simplest function in terms of which we can express the Coulomb interaction energy,

$$
V_{e e}[I]=\int d s \frac{I\left(\rho_{2}, s\right)}{s} .
$$


The electron correlation contents of the pair density can be determined by the difference between the actual pair density and an uncorrelated reference, which here we choose to be the Hartree-Fock (HF) one,

$$
\Delta \rho_{2}^{\mathrm{c}}(\mathbf{1}, \mathbf{2})=\rho_{2}(\mathbf{1}, \mathbf{2})-\rho_{2}^{\mathrm{HF}}(\mathbf{1}, \mathbf{2}) .
$$

The intracule of this function is Coulson's Coulomb hole, ${ }^{29}$

$$
h_{\mathrm{c}}(s)=I\left(\Delta \rho_{2}^{\mathrm{c}}, s\right)=\int d_{1} d_{2} \Delta \rho_{2}^{\mathrm{c}}(\mathbf{1}, \mathbf{2}) \delta\left(s-r_{12}\right) .
$$

In order to split the correlation part of the pair density (Eq. 4) we employ an approximate pair density, the single-determinant (SD) ansatz of the pair density, ${ }^{1}$

$$
\rho_{2}^{\mathrm{SD}}\left(\rho_{1}, \mathbf{1}, \mathbf{2}\right)=\rho_{1}(\mathbf{1}) \rho_{1}(\mathbf{2})-\left|\rho_{1}(\mathbf{1} ; \mathbf{2})\right|^{2},
$$

where $\rho_{1}(\mathbf{1} ; \mathbf{2})$ is the $1-\mathrm{RDM}$ and $\rho_{1}(\mathbf{1}) \equiv \rho_{1}(\mathbf{1} ; \mathbf{1})$ is the electron density. Substituting $\rho_{1}$ by the HF 1-RDM in Eq. 6, yields the HF pair density, i.e.,

$$
\rho_{2}^{\mathrm{HF}}(\mathbf{1}, \mathbf{2})=\rho_{2}^{\mathrm{SD}}\left(\rho_{1}^{\mathrm{HF}}, \mathbf{1}, \mathbf{2}\right),
$$

which does not account for electron correlation. However, $\rho_{2}^{\mathrm{SD}}\left(\rho_{1}, \mathbf{1}, \mathbf{2}\right)$ can be regarded as an approximation to the actual pair density; an approximation which does not account for dynamic correlation either at short ${ }^{30}$ or at long range. ${ }^{28}$ Figure 1 depicts the two paths of arriving at the exact $\rho_{2}\left(\rho_{1}, \mathbf{1}, \mathbf{2}\right)$ from $\rho_{2}^{\mathrm{SD}}\left(\rho_{1}^{\mathrm{HF}}, \mathbf{1}, \mathbf{2}\right)$, either straightforwardly or through the intermediate SD approximation. The latter path defines the decomposition of the correlation part of the pair density,

$$
\begin{aligned}
\Delta \rho_{2}^{\mathrm{c}}(\mathbf{1}, \mathbf{2}) & =\left(\rho_{2}\left(\rho_{1}, \mathbf{1}, \mathbf{2}\right)-\rho_{2}^{\mathrm{SD}}\left(\rho_{1}, \mathbf{1}, \mathbf{2}\right)\right)+\left(\rho_{2}^{\mathrm{SD}}\left(\rho_{1}, \mathbf{1}, \mathbf{2}\right)-\rho_{2}^{\mathrm{SD}}\left(\rho_{1}^{\mathrm{HF}}, \mathbf{1}, \mathbf{2}\right)\right) \\
& =\Delta \rho_{2}^{\mathrm{c}_{I}}(\mathbf{1}, \mathbf{2})+\Delta \rho_{2}^{\mathrm{c}_{I I}}(\mathbf{1}, \mathbf{2}) .
\end{aligned}
$$

$\Delta \rho_{2}^{c_{I}}(\mathbf{1}, \mathbf{2})$ will be large only if the HF 1-RDM and the actual 1-RDM are significantly different 


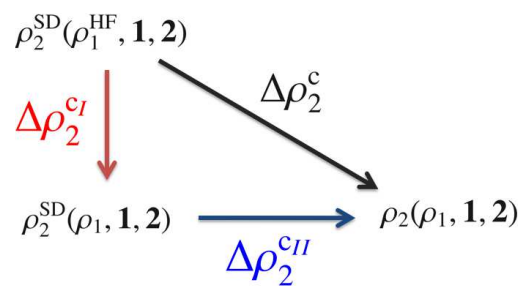

Figure 1: The correlation part of the pair density, $\Delta \rho_{2}^{\mathrm{c}}$, decomposed into two components.

and, in such case, the system will be affected by nondynamic correlation. Indeed, the wavefunction of systems dominated by dynamic correlation can be described by a large expansion of Slater determinants with one of them (the HF one) having an expansion coefficient very close to one. ${ }^{31}$ Therefore, these systems are characterized by a 1-RDM that retains the shape of the HF 1-RDM. Conversely, the wavefunction of nondynamic-correlated systems can be written as a shorter expansion of Slater determinants, but in this case the HF determinant has an expansion coefficient that is qualitatively smaller than one. ${ }^{31}$ Since the $1-\mathrm{RDM}$ is determined by the square of the expansion coefficients, we expect systems affected by nondynamic correlation to display large $\Delta \rho_{2}^{\mathrm{c}_{I}}(\mathbf{1}, \mathbf{2})$. Some authors have used similar arguments to use the electron density (the diagonal part of the 1-RDM) as a means to define dynamic and nondynamic correlation energy. ${ }^{2,6}$ In this work, we prefer to employ the 1-RDM because the cases of spin entaglement would not be regarded as nondynamic correlation if only density differences were considered. Indeed, in the stretched $\mathrm{H}_{2}$ molecule, the $\mathrm{HF}$ electron density is qualitatively similar to the exact one, whereas there are large and notorious differences between the exact and the HF 1-RDMs.

The magnitude of $\Delta \rho_{2}^{\mathrm{c}_{I}}(\mathbf{1}, \mathbf{2})$ can be thus regarded as a measure of nondynamic correlation but it can also be interpreted as the correlation retrieved by using the actual 1-RDM rather than the HF one to construct the pair density. Conversely, $\Delta \rho_{2}^{c_{I I}}(\mathbf{1}, \mathbf{2})$ does not depend on the differences between $\rho_{1}^{\mathrm{HF}}$ and $\rho_{1}$, but on the validity of the SD approximation. Note that $\Delta \rho_{2}^{\mathrm{c}_{I I}}$ coincides with the cumulant of the pair density. ${ }^{14,32}$ The intracule functions of $\rho_{2}^{\mathrm{SD}}\left(\rho_{1}, \mathbf{1}, \mathbf{2}\right)$ and the exact pair density, $\rho_{2}\left(\rho_{1}, \mathbf{1}, \mathbf{2}\right)$, display the same asymptotic behavior ${ }^{33}$ and, therefore, $\Delta \rho_{2}^{\mathrm{c}_{I I}}$ is dominated by the short-range component. Interestingly, $\Delta \rho_{2}^{\mathrm{c}_{I}}(\mathbf{1}, \mathbf{2})$ is the long-range-dominant component of the 
correlated part of the pair density (Eq. 4) because the HF and the exact 1-RDM can differ substan-

tially at large separations, for instance, in the presence of entanglement. On the contrary, $\Delta \rho_{2}^{\mathrm{c}_{I}}(\mathbf{1}, \mathbf{2})$ displays very small values at small interelectronic distances mostly due to the opposite-spin part of this term.

The current partition,

$$
\rho_{2}(\mathbf{1}, \mathbf{2})=\rho_{2}^{\mathrm{HF}}(\mathbf{1}, \mathbf{2})+\Delta \rho_{2}^{\mathrm{c}_{I}}(\mathbf{1}, \mathbf{2})+\Delta \rho_{2}^{\mathrm{c}_{I I}}(\mathbf{1}, \mathbf{2})
$$

provides a natural range separation of the pair density that can be employed to split the Coulomb hole into two correlation components,

$$
h_{\mathrm{c}}(s)=h_{\mathrm{c}_{I}}(s)+h_{\mathrm{c}_{I I}}(s)=I\left(\Delta \rho_{2}^{\mathrm{c}_{I}}, s\right)+I\left(\Delta \rho_{2}^{\mathrm{c}_{I I}}, s\right) \text {, }
$$

naturally yielding a separation of electron correlation by range. We will show that the decay of $I\left(\Delta \rho_{2}^{\mathrm{c}_{I I}}, R\right)$ is universal and it corresponds to a characteristic signature of London dispersion forces ( $R$ being the distance between two atoms in the molecule).

\section{Results and Discussion}

In the following we introduce five selected examples that illustrate the effectiveness of the current scheme to separate the correlation part of the Coulomb hole at different ranges and how the longrange of $\Delta \rho_{2}^{\mathrm{c}_{I I}}$ can be used to identify and characterize van der Waals interactions.

The Hydrogen Molecule ${ }^{34}$. - At the equilibrium geometry, $h_{\mathrm{c}_{I I}}(s)$ dominates over $h_{\mathrm{c}_{I}}(s)$ at all interelectronic distances $s$, as shown in the left panel of Fig. 2, whereas $h_{\mathrm{c}_{I}}(s)$ increases importantly as the bond is stretched, in line with the expected increase of nondynamic correlation. The most likely distribution of the electron pair at large bond lengths corresponds to one electron sitting at each atom and, accordingly, the intracule density peaks around the bond-length distance. At the 
dissociation limit, the long-range part of the Coulomb hole is completely determined by $h_{\mathrm{c}_{I}}(s)$ because one isolated electron cannot give rise to dynamic correlation. Hence, the unrestricted HF calculation of $\mathrm{H}_{2}$ produces Coulomb hole components that are not distinguishable from FCI. ${ }^{35}$ A simple interpretation is also obtained from valence bond theory: at large separations, the exact pair density is entirely described by covalent components, whereas the HF pair density contains equally contributing ionic and covalent terms. $h_{\mathrm{c}_{I I}}(s)$ removes the ionic contribution (i.e., removes contributions keeping the electrons at short distances), whereas $h_{\mathrm{c}_{I}}(s)$ adds the missing covalent contribution (i.e., adds contributions placing one electron in each atom); in accord with the results plotted in the r.h.s. of Fig. 2 (see also Supp. Material).
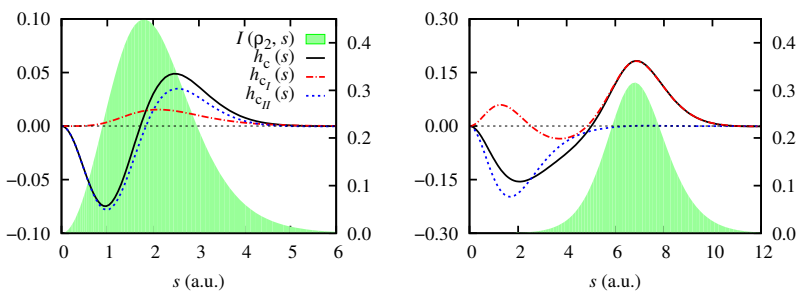

Figure 2: The total Coulomb hole (black), $h_{\mathrm{c}_{I}}(s)$ (red) and $h_{\mathrm{c}_{I I}}(s)$ (blue) correlation components, and the intracule density (shadowed green region, right $y$-axis) of the $\mathrm{H}_{2}$ molecule at 1.32 and 7.56 a.u. bond lengths.

The Hubbard Dimer. - The Hubbard dimer is the simplest model of interacting particles in a lattice and conceivably the most studied model for testing methods at different regimes. ${ }^{36,37} \mathrm{We}$ employ the one-dimension Hamiltonian of the Hubbard model,

$$
\hat{H}=-t \sum_{\langle\mu, v\rangle, \sigma}\left(\hat{c}_{\mu \sigma}^{\dagger} \hat{c}_{\nu \sigma}+\hat{c}_{\nu \sigma}^{\dagger} \hat{c}_{\mu \sigma}\right)+U \sum_{\mu} \hat{\rho}_{\mu \alpha} \hat{\rho}_{\mu \beta}
$$

where $\mu$ and $v$ denote the sites, $\sigma$ the spin polarization $\left(\alpha\right.$ or $\beta$ ), $\hat{c}_{\mu \sigma}^{\dagger}$ and $\hat{c}_{\mu \sigma}$ are creation and annihilation operators of one electron with spin $\sigma$ in site $\mu$, and $\hat{\rho}_{\mu \sigma}$ stands for a one-particle number operator with spin $\sigma$ acting on site $\mu$. $t$ is the hopping parameter and $U$ is the on-site interaction parameter. These parameters control the electron correlation within the Hubbard model, small (large) $U / t$ inducing dynamic (nondynamic) correlation. Hence large $U / t$ values prompt the electrons to distribute among the sites to minimize the electron repulsion. Fig. 3 presents plots of 
the Coulomb hole at various values of $U / t$ for the two-electron two-site Hubbard model in real space. ${ }^{36}$ At low $U / t$ values, the system is barely affected by correlation, thus dynamic correlation dominates (small $h_{\mathrm{c}_{I}}(s)$ and large $h_{\mathrm{c}_{I I}}(s)$ ) and the electron pairs distribute equally between on-site and intersite components. As $U / t$ grows, nondynamic correlation dominates and $h_{\mathrm{c}_{I}}(s)$ becomes more important, being the prevailing contribution between sites.
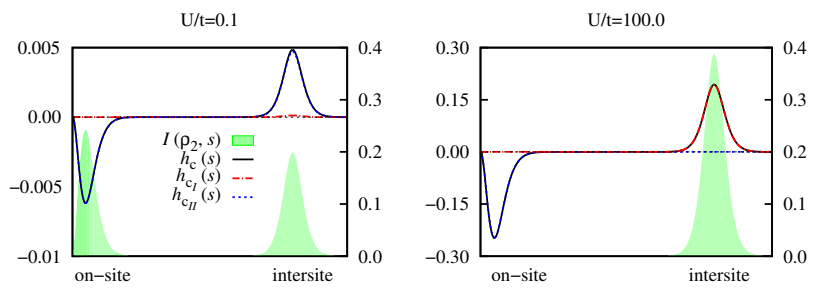

Figure 3: The Coulomb hole (see Fig. 2 for further details) of the two-site real-space Hubbard model for various $U / t$ values.

The He series. ${ }^{34}$ - The He isoelectronic series is perhaps the simplest series of systems dominated by dynamic correction. ${ }^{38}$ As the atomic number $Z$ increases, the electron correlation of $\mathrm{He}(Z)$ tends to a constant and the exact electron density barely distinguishes from the HF one. In Fig. 4 we observe that $h_{\mathrm{c}_{I}}(s)$ decreases with the atomic number $Z$ and, hence, $h_{\mathrm{c}_{I I}}$ completely takes over.
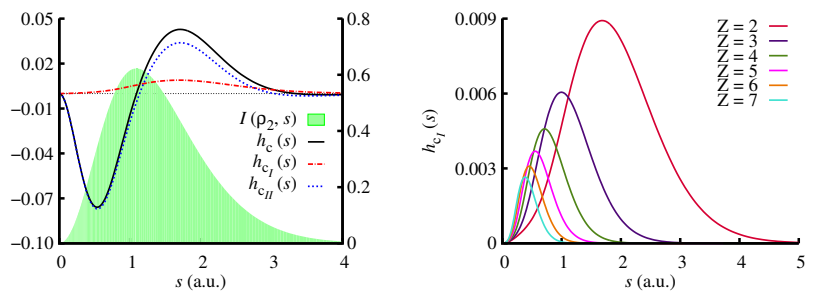

Figure 4: The Coulomb hole of He (1.h.s.) and $h_{\mathrm{c}_{I}}(s)$ for the isoelectronic series of He (r.h.s.).

$N$ hydrogen atoms. ${ }^{34}$ — The size consistency of our approach and its ability to measure spin entanglement is examined in Fig. 5. We have plotted the Coulomb hole of the $N$-vertex polyhedron resulting from $N$ hydrogen atoms separated by $10 \AA$ from the center of the polyhedron. At these large separations, the hydrogen atoms only interact to each other through entanglement and this is the only term that remains in the cumulant, ${ }^{4}$ (i.e. in $\Delta \rho_{2}^{\mathrm{c} I I}$ ), which shows a linear behavior with 
$N$ (see Fig. 5). As in previous systems, $h_{\mathrm{c}_{I I}}$ is short ranged and its contribution to the energy grows linearly with $N$. These systems can be classified as nondynamic correlated because $h_{\mathrm{c}_{I}}$ is mostly long ranged and peaks at the same positions of the intracule density maxima. The planar $D_{4 h} / D_{2 h}$ potential energy surface of $\mathrm{H}_{4}$ has also been used for discriminating between dynamic and nondynamic correlation ${ }^{39}$ and is given in the Supp. Material.
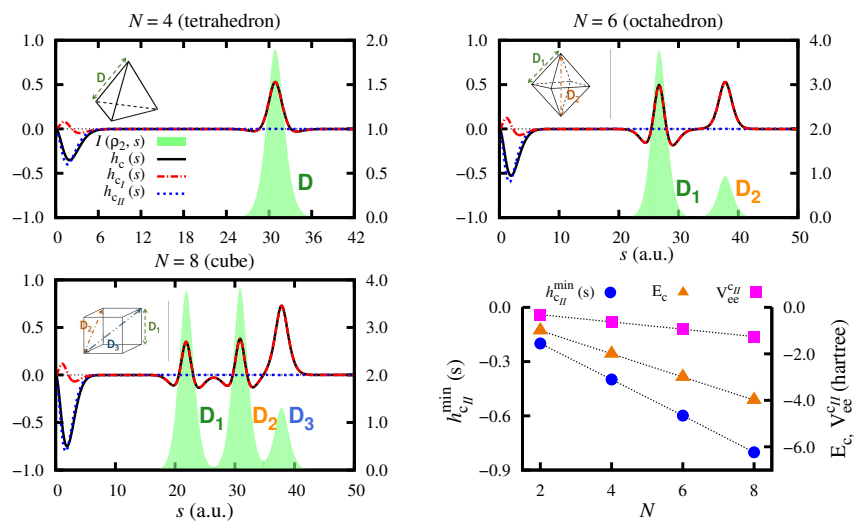

Figure 5: The Coulomb hole of $N$ hydrogen atoms. $\mathrm{D}, \mathrm{D}_{1}, \mathrm{D}_{2}$, and $\mathrm{D}_{3}$ indicate the different distances between the $\mathrm{H}$ atoms placed at the vertices of the respective polyhedra. The bottom r.h.s. plot displays the minimal value of $h_{\mathrm{c}_{I I}}, h_{\mathrm{c}_{I I}}^{\min }(s)$, the part of electron-electron repulsion that corresponds to $\Delta \rho_{2}^{\mathrm{c}_{I I}}\left(\right.$ i.e. $\left.V_{e e}^{\mathrm{c}_{I I}}=\int d s h_{\mathrm{c}_{I I}}(s) / s\right)$, and the correlation energy $\left(\mathrm{E}_{c}\right)$, as a function of $N$.

van der Waals (vdW) Interactions. ${ }^{34}$ - Fig. 6 includes plots of the Coulomb hole of the helium dimer. $h_{c}$ compares satisfactorily to earlier calculations. ${ }^{40}$ The dynamic long-range interaction between the two noble-gas atoms is reflected by the second peak of the intracule density, whereas the interaction of the electron pair within each helium shows in the first peak. Regardless the bond length, $h_{\mathrm{c}_{I I}}$ dominates, indicating that the correlation is dynamic and mainly affects the electron pair within each $\mathrm{He}$. Unlike $\mathrm{H}_{2}$, there is very little long-range nondynamic correlation in this system; however, at all distances, the long-range part of $h_{\mathrm{c}_{I I}}$ peaks around the bond-length distance (see the inset plots of Fig. 6). The plot in Fig. 7 presents $h_{\mathrm{c}_{I I}}(R)$ against the bond length, $R$, revealing a $R^{-3}$ decay. It is a textbook fact that the pairwise vdW energy decays like $R^{-6} \cdot{ }^{41}$ Using perturbation theory, we have recently proved that the vdW contribution to $h_{\mathrm{c}_{I I}}$ should actually decay like $R^{-3}$, the integration of $h_{\mathrm{c}_{I I}}(s) / s$ over $s$ yielding a fraction of the Coulombic interaction (Eq. 3) due to London dispersion forces and, therefore, decaying as $R^{-6} \cdot{ }^{28}$ Fig. 7 includes plots for other noble- 
gas dimers, which also satisfy this property. Most density functional theory (DFT) practitioners add ad hoc empirical corrections to the energy for vdW interactions and, therefore, they only shift the relative energies of different conformers, yet the electronic structure of the system is not completely considered. ${ }^{42}$ The present separation into correlation regimes unveils the target part of the pair density and the Coulomb hole, i.e., the long-range component of $h_{\mathrm{c}_{I I}}(s)$, which should be improved in order to incorporate the description of London dispersion forces and avoid the latter problem, thus opening a door to the accurate account of these forces within DFT and reduced density matrix functional theory (RDMFT).
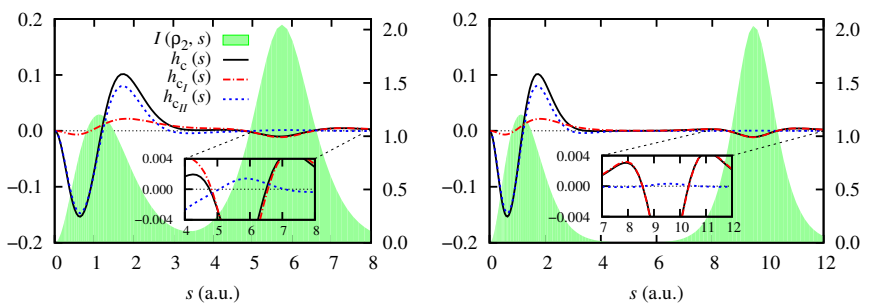

Figure 6: The Coulomb hole of the $\mathrm{He}_{2}$ molecule at two bond lengths (5.67 and 9.45 a.u., left and right). The inset plots reproduce the ones above on a narrower interval.
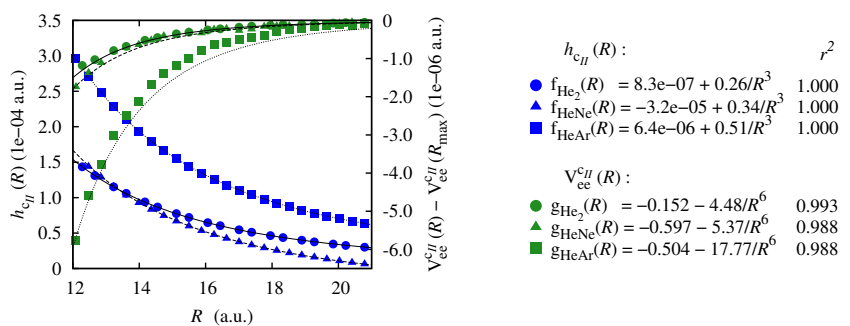

Figure 7: $h_{\mathrm{c}_{I I}}(R)$ (in blue) against the bond length $(R)$ and the corresponding electron-electron van der Waals contributions (in green) for several noble-gas dimers: $\mathrm{He}_{2}, \mathrm{HeNe}$ and $\mathrm{HeAr}$.

In conclusion, Eqs. 8 and 9 represent a separation of the pair density and the Coulomb hole into components dominated by short- and long-range interactions. This result is expected to be important in the development of new hybrid electronic structure methods that can be employed in RDMFT $^{21,43}$ and other computational approaches. For instance, the HF reference in Fig. 1 can be replaced by the Kohn-Sham system to adapt the present idea to DFT. It can be shown that the exchange-correlation functional can be entirely written in terms of the Kohn-Sham orbitals, $\Delta \rho_{2}^{\mathrm{c}_{I}}$, 
and $\Delta \rho_{2}^{\mathrm{c}_{I I}}$. Hence, a template to construct density functional approximations, where the correlation components are treated separately, arises. Such possibility is already being explored in our laboratory.

\section{Acknowledgements}

We thank D. Casanova, X. Lopez, P. Salvador, and specially, P.M.W. Gill and J.M. Ugalde for helpful insights. This research has been funded by Spanish MINECO/FEDER Projects CTQ201452525-P, PGC2018-098212-B-C21, and EUIN2017-88605. We acknowledge doctoral grants BES2015-072734 and FPU-2013/00176, and the funding from the European Union's Horizon 2020 research and innovation programme under the Marie Sklodowska-Curie grant agreement (No. 660943).

Supporting Information Available: Analysis of the $D_{2 h} / D_{4 h} \mathrm{H}_{4}$ planar molecule and the hydrogen molecule from ionic and covalent contributions. Full plots of $\mathrm{H}_{2}$, the two-site Hubbard dimer, the He isoelectronic series, and the $N$ hydrogen atoms model.

\section{References}

(1) Löwdin, P.-O. Quantum theory of many-particle systems. I. Physical interpretations by means of density matrices, natural spin-orbitals, and convergence problems in the method of configurational interaction. Phys. Rev. 1955, 97, 1474-1489.

(2) Cioslowski, J. Density-driven self-consistent-field method: Density-constrained correlation energies in the helium series. Phys. Rev. A 1991, 43, 1223-1228.

(3) Gottlieb, A. D.; Mauser, N. J. New measure of electron correlation. Phys. Rev. Lett. 2005, 95, 123003. 
(4) Raeber, A.; Mazziotti, D. A. Large eigenvalue of the cumulant part of the two-electron reduced density matrix as a measure of off-diagonal long-range order. Phys. Rev. A 2015, 92, 052502.

(5) Benavides-Riveros, C. L.; Lathiotakis, N. N.; Schilling, C.; Marques, M. A. Relating correlation measures: The importance of the energy gap. Phys. Rev. A 2017, 95, 032507.

(6) Valderrama, E.; Ludeña, E. V.; Hinze, J. Analysis of dynamical and nondynamical components of electron correlation energy by means of local-scaling density-functional theory. $J$. Chem. Phys. 1997, 106, 9227-9235.

(7) Valderrama, E.; Ludeña, E. V.; Hinze, J. Assessment of dynamical and nondynamical correlation energy components for the beryllium-atom isoelectronic sequence. J. Chem. Phys. 1999, 110, 2343-2353.

(8) Mok, D. K. W.; Neumann, R.; Handy, N. C. Dynamic and Nondynamic Correlation. J. Phys. Chem. 1996, 100, 6225-6230.

(9) Benavides-Riveros, C. L.; Lathiotakis, N. N.; Marques, M. A. Towards a formal definition of static and dynamic electronic correlations. Phys. Chem. Chem. Phys. 2017, 19, 12655-12664.

(10) Ziesche, P. On relations between correlation, fluctuation and localization. J. Mol. Struct. (Theochem) 2000, 527, 35-50.

(11) Ramos-Cordoba, E.; Salvador, P.; Matito, E. Separation of dynamic and nondynamic correlation. Phys. Chem. Chem. Phys. 2016, 18, 24015-24023.

(12) Ramos-Cordoba, E.; Matito, E. Local Descriptors of dynamic and nondynamic correlation. J. Chem. Theory Comput. 2017, 13, 2705-2711.

(13) Juhász, T.; Mazziotti, D. A. The cumulant two-particle reduced density matrix as a measure of electron correlation and entanglement. J. Chem. Phys. 2006, 125, 174105. 
(14) Mazziotti, D. A. Approximate solution for electron correlation through the use of Schwinger probes. Chem. Phys. Lett. 1998, 289, 419-427.

(15) Ramos-Cordoba, E.; Salvador, P.; Piris, M.; Matito, E. Two new constraints for the cumulant matrix. J. Chem. Phys. 2014, 141, 234101.

(16) Cioslowski, J.; Piris, M.; Matito, E. Robust validation of approximate 1-matrix functionals with few-electron harmonium atoms. J. Chem. Phys. 2015, 143, 214101.

(17) Pastorczak, E.; Shen, J.; Hapka, M.; Piecuch, P.; Pernal, K. Intricacies of van der Waals interactions in systems with elongated bonds revealed by electron-groups embedding and high-level coupled-cluster approaches. J. Chem. Theory Comput. 2017, 13, 5404-5419.

(18) Savin, A. A combined density functional and configuration interaction method. Int. J. Quantum Chem. 1988, 34, 59-69.

(19) Grimme, S.; Waletzke, M. A combination of Kohn-Sham density functional theory and multireference configuration interaction methods. J. Chem. Phys. 1999, 111, 5645-5655.

(20) Bao, J. J.; Gagliardi, L.; Truhlar, D. G. Multiconfiguration pair-density functional theory for doublet excitation energies and excited state geometries: the excited states of CN. Phys. Chem. Chem. Phys. 2017, 19, 30089-30096.

(21) Piris, M. Global Method For The Electron Correlation. Phys. Rev. Lett. 2017, 119, 063002.

(22) Iikura, H.; Tsuneda, T.; Yanai, T.; Hirao, K. A long-range correction scheme for generalizedgradient-approximation exchange functionals. J. Chem. Phys. 2001, 115, 3540-3544.

(23) Toulouse, J.; Gerber, I. C.; Jansen, G.; Savin, A.; Angyán, J. G. Adiabatic-connection fluctuation-dissipation density-functional theory based on range separation. Phys. Rev. Lett. 2009, 102, 096404.

(24) Toulouse, J.; Colonna, F.; Savin, A. Long-range short-range separation of the electronelectron interaction in density-functional theory. Phys. Rev. A 2004, 70, 062505. 
(25) Baer, R.; Livshits, E.; Salzner, U. Tuned range-separated hybrids in density functional theory. Ann. Rev. Phys. Chem. 2010, 61, 85-109.

(26) Garrett, K.; Sosa Vazquez, X.; Egri, S. B.; Wilmer, J.; Johnson, L. E.; Robinson, B. H.; Isborn, C. M. Optimum exchange for calculation of excitation energies and hyperpolarizabilities of organic electro-optic chromophores. J. Chem. Theory Comput. 2014, 10, 3821-3831.

(27) Vuckovic, S.; Irons, T. J. P.; Wagner, L. O.; Teale, A. M.; Gori-Giorgi, P. Interpolated energy densities, correlation indicators and lower bounds from approximations to the strong coupling limit of DFT. Phys. Chem. Chem. Phys. 2017, 19, 6169-6183.

(28) Via-Nadal, M.; Rodríguez-Mayorga, M.; Matito, E. A Salient Signature of van der Waals Interactions. Phys. Rev. A 2017, 96, 050501.

(29) Coulson, C. A.; Neilson, A. H. Electron correlation in the ground state of helium. Proc. Phys. Soc. London 1961, 78, 831-837.

(30) Rodríguez-Mayorga, M.; Ramos-Cordoba, E.; Via-Nadal, M.; Piris, M.; Matito, E. Comprehensive benchmarking of density matrix functional approximations. Phys. Chem. Chem. Phys. 2017, 19, 24029-24041.

(31) Rigorously speaking, the condition that the coefficients should satisfy is that their $\mathrm{N}^{\text {th }}$ root, $N$ being the number of electrons, is close to one for the system to be dominated by dynamic correlation. Otherwise, we would not correctly consider as such composites of noninteracting dynamic-correlation-driven systems, e.g., an infinite number of non-interacting helium atoms.

(32) Kutzelnigg, W.; Mukherjee, D. Cumulant expansion of the reduced density matrices. J. Chem. Phys. 1999, 110, 2800-2809.

(33) Ernzerhof, M.; Burke, K.; Perdew, J. P. Long-range asymptotic behavior of ground-state wave functions, one-matrices, and pair densities. J. Chem. Phys. 1996, 105, 2798-2803. 
(34) We have performed full configuration interaction (FCI) calculations with the aug-cc-pVDZ basis set for $\mathrm{He}_{2}$ and $\mathrm{H}_{n}(n=2-8)$, and single and double configuration interactions calculations with the aug-cc-pVTZ basis set for HeNe and HeAr molecules. For the isoelectronic series of He we have used an even-tempered basis set of $5 \mathrm{~s}, 5 \mathrm{p}$ and $5 \mathrm{~d}$ functions, optimized following the procedure described elsewhere ${ }^{44}$.

(35) Mercero, J. M.; Valderrama, E.; Ugalde, J. M. In Metal-Ligand Interactions; Russo, N., Salahub, D. R., Witko, M., Eds.; Kluwer Academic Publishers: The Netherlands, 2003; pp 205-239.

(36) Carrascal, D.; Ferrer, J.; Smith, J. C.; Burke, K. The Hubbard dimer: a density functional case study of a many-body problem. J. Phys.: Condens. Matter 2015, 27, 393001.

(37) Deur, K.; Mazouin, L.; Fromager, E. Exact ensemble density functional theory for excited states in a model system: Investigating the weight dependence of the correlation energy. Phys. Rev. B 2017, 95, 035120.

(38) Chakravorty, S. J.; Gwaltney, S. R.; Davidson, E. R.; Parpia, F. A.; p Fischer, C. F. Groundstate correlation energies for atomic ions with 3 to 18 electrons. Phys. Rev. A 1993, 47, 3649.

(39) Ramos-Cordoba, E.; Lopez, X.; Piris, M.; Matito, E. $\mathrm{H}_{4}$ : A challenging system for natural orbital functional approximations. J. Chem. Phys. 2015, 143, 164112.

(40) Piris, M.; Lopez, X.; Ugalde, J. Correlation holes for the helium dimer. J. Chem. Phys. 2008, $128,134102$.

(41) Pauling, L.; Wilson, E. B. Introduction to quantum mechanics; Dover Publications, Inc.: New York, 1935.

(42) Hermann, J.; DiStasio Jr, R. A.; Tkatchenko, A. First-Principles Models for van der Waals Interactions in Molecules and Materials: Concepts, Theory, and Applications. Chem. Rev. 2017, 117, 4714-4758. 
(43) Piris, M.; Ugalde, J. M. Perspective on natural orbital functional theory. Int. J. Quantum Chem. 2014, 114, 1169-1175.

(44) Matito, E.; Cioslowski, J.; Vyboishchikov, S. F. Properties of harmonium atoms from FCI calculations: Calibration and benchmarks for the ground state of the two-electron species. Phys. Chem. Chem. Phys. 2010, 12, 6712. 